\title{
Design, Integration and Characterization of a Novel Paper-based Wireless Sensor Module
}

\author{
Rushi Vyas, Amin Rida, Li Yang and Manos M. Tentzeris \\ Georgia Electronic Design Center, School of Electrical and Computer Engineering, \\ Georgia Institute of Technology, Atlanta, GA 30332-0250, USA \\ E-mail: Rushi.Vyas@gatech.edu
}

\begin{abstract}
In this paper, the first ever wireless "System On Paper" is introduced. A paper based wireless sensor module verifies the feasibility of this technology. Inkjet printing of conductive nanoparticle silver ink, as well as the efficient integration of sensor active and passive devices and power source, reported in this paper, set the foundation for the development of low-cost light-weight autonomous nodes for cognitive intelligence applications.
\end{abstract}

\section{INTRODUCTION}

As the demand for low-cost, flexible and power-efficient broadband wireless electronics increase, the materials and integration techniques become more and more critical and face more challenges, especially with the ever increasing interest for "cognitive intelligence" and "ubiquitous wireless networks". This paper introduces the FIRST EVER wireless sensor module built and integrated COMPLETELY on paper. Specifically, a paper-based microcontroller enabled wireless sensor prototype has been developed for the first time for the UHF frequency band (centered at $904.4 \mathrm{MHz}$ ).

Paper is considered one of the best organic-substrate candidates for UHF and microwave applications for many reasons. It features the industrial ability to be processed in a reel-to-reel fashion. Paper can also make utilization of direct write methodologies such as inkjet-printing, to be used in replacement of relatively expensive wet-etching techniques. Such a fast process can be used to print electronics on the surface of paper substrate or even embedded in a multilayer fashion. Last, but not least, paper is one of the most environmentally-friendly materials and the proposed module could potentially set the foundation for the first generation of truly "green" RF electronics and modules.

\section{SYSTEM LEVEL DESIGN}

To investigate the feasibility of paper as an RF frequency substrate and packaging material, a microcontroller enabled wireless sensor module was realized on a photo-paper-based substrate for the first time. The system level design for this wireless transmitter can be seen in fig. 1 below. At the heart of the unit was an integrated 8-bit integrated microcontroller unit (MCU) that was programmed to sample an analog temperature sensor, perform an analog to digital conversion of the sensed data, bit encode the digital form of the sensed data into full 2 sub carrier cycle Miller bits [2] and finally modulate the power amplifier in the integrated transmitter module in the same sequence as bit encoded, digital sensor data using ASK modulation. The transmission frequency of $904.4 \mathrm{MHz}$ was generated by using a crystal oscillator that was tied to the input of the PLL unit of the transmitter. The data transmission was to be carried out at the unlicensed UHF frequencies around $900 \mathrm{MHz}$. The entire wireless system including the antenna was to be printed on a 2-D paper module. The full design process will be outlined in this section.

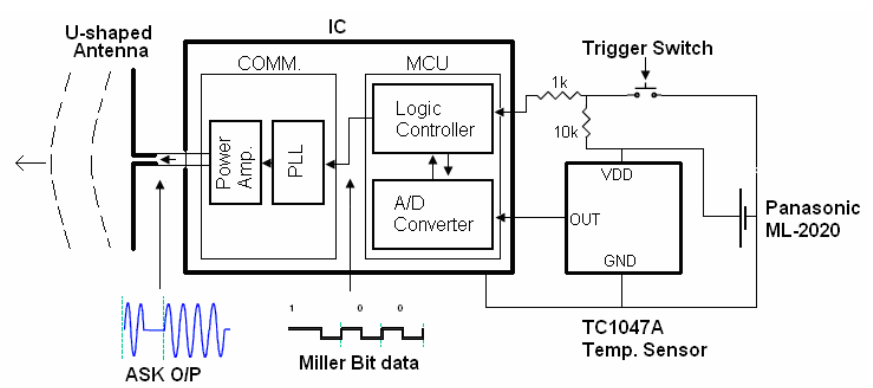

Fig 1. System level diagram of wireless sensor module.

\section{A. Amplifier Characterization}

The range of the wireless transfer is directly proportional to the amount of power transferred from the amplifier in the transmitter front end to the antenna. Any impedance mismatch between the two can lead to the reflection of a part of the power intended to radiate out of the antenna thereby minimizing range. Instead of designing the antenna to a 50 match, it was decided to design it to the optimum impedance looking out of the amplifier in the transmitter. Doing so would eliminate the need for a matching network minimizing series losses as well as components used in the design, thus leading to a more compact design. To determine the optimum load impedance looking out of the amplifier in the transmitter, a load pull analysis was performed on it at frequencies of 860, 904.4 and $925 \mathrm{MHz}$ for a fixed bias point. The setup used for this measurement is shown in figure 2. For this measurement, a prototype of the entire system was implemented on a Copper-clad Duroid board. The output of the transmitter was interfaced to the measurement setup using a CPW and an SMA on the prototype board. Interfaces were also provided to 
the IC on the prototype board to enable in-circuit programming and debugging of the Microcontroller unit in it shown in figure 3.

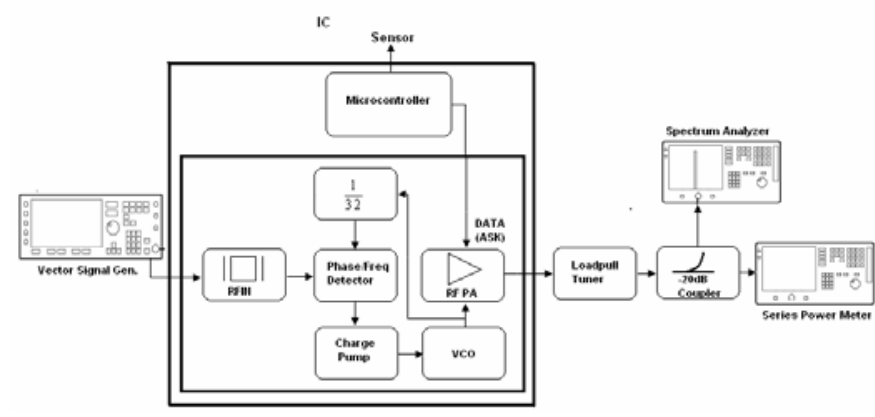

Fig. 2. Load-pull setup for Amplifier Characterization.

For the load-pull measurements, the microcontroller was programmed to maintain a constant "ON" voltage at the Data/ASK pin of the transmitter shown in figure 2 thereby sending a constant unmodulated sinusoidal signal at the transmitter output. The loss in the measurement setup i.e. the RF tuner, the $-20 \mathrm{~dB}$ coupler and the cables were measured separately at each of the three measurement frequencies and added to the power output measured with the power meter to get the optimum output power shown in Table 1. The effects of the SMA connector, CPW feed, the series capacitor and bias circuitry were de-embedded to get the optimum load impedance at the point looking out of the amplifier in the IC. The impedances were de-embedded using Agilent's Advanced Design System (ADS) software and are shown in table 1.

Table 1. Load Pull measurements for Power Amplifier characterization.

\begin{tabular}{|l|l|l|l|}
\hline $\begin{array}{l}\text { Freq. } \\
(\mathrm{MHz})\end{array}$ & $\mathrm{Z}_{\mathrm{L}-\mathrm{ppt}}$ at IC ( ) & $\begin{array}{l}\text { Output Power- } \\
\text { Measured } \\
(\mathrm{dBm})\end{array}$ & $\begin{array}{l}\text { Output Power- } \\
\text { Manufacturer. } \\
(\mathrm{dBm})\end{array}$ \\
\hline 860 & $117.5+\mathrm{j} 137.14$ & 6.1 & 7.5 \\
\hline 904.4 & $60.1-\mathrm{j} 73.51$ & 7.43 & 7.5 \\
\hline 925 & $35.9-\mathrm{j} 15.87$ & 7.72 & 7.5 \\
\hline
\end{tabular}

An Agilent E4438C VSG was used to generate the input RF signal, while the output power was measured using the Agilent E4419B RPM power meter. A portion of the output power was coupled to an Agilent E4404B spectrum analyzer to ensure that the power meter was measuring the output power at the correct output RF frequency. The RF tuner used was a Maury model 1643-N. The optimum impedance values obtained using this method were then used to design a compact wideband antenna. The center frequency chosen was 904.4 MHz to be safely within the unlicensed UHF spectrum.

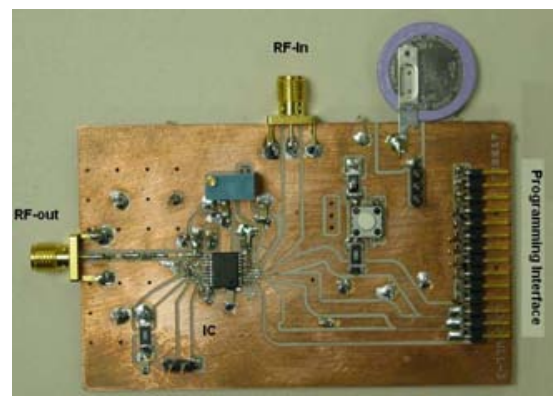

Fig. 3. Prototyping board for load-pull analysis and in-circuit programming.

\section{B. Antenna Design}

The antenna used for this design was designed using Ansoft's HFSS 3-D EM solver. The antenna shape chosen was a U-shaped tapered dipole [1] to reduce the overall size of the antenna while at the same time achieving a wide bandwidth near the design center frequency. The U-shape tapered structure shown in fig. 4 is the antenna. The antenna features impedance around the resonance frequency that is matched to the value of $37+\mathrm{j} 22 \mathrm{Ohms}$ at $904.4 \mathrm{MHz}$ as mentioned in table 1. This impedance was determined after accounting for the shunt impedance introduced by the bias circuit and the series impedance introduced by the series coupling capacitor between the amplifier $\left(Z_{\mathrm{LOPT}}\right.$ as shown in table 1$)$ and the antenna terminals at $904.4 \mathrm{MHz}$. The passive components used in this module had self resonance frequencies close to the transmission frequency; their effective impedances were measured and de-embedded using a Vector Network Analyzer VNA (Rohde and Schwarz ZVA-8) at the frequency of interest The $\lambda / 2$ antenna was folded in a U-shape at a distance from the center $\quad(\sim 0.08 \lambda$, where $\lambda$ corresponds to the wavelength at the design frequency), such that it would not cause any significant current perturbation thus maintaining the maximum radiation efficiency, while fitting all of the electronic components within the subtended area of the Ushape antenna, hence making the design more compact. The overall dimensions of the structure are: $9.5 \times 5 \mathrm{~cm}$.

The Return Loss or S11 recordings for the center frequency for the antenna terminals were recorded to be $-15.05 \mathrm{~dB}$ for the simulated structure using the full wave EM simulator HFSS and $-12.45 \mathrm{~dB}$ measured using the ZVA-8 VNA mentioned above. The radiation pattern was also measured using Satimo's Stargate 64 Antenna Chamber measurement system and by using the NIST Calibrated SH8000 Horn Antenna as a calibration kit for the measured radiation pattern at $904 \mathrm{MHz}$. 


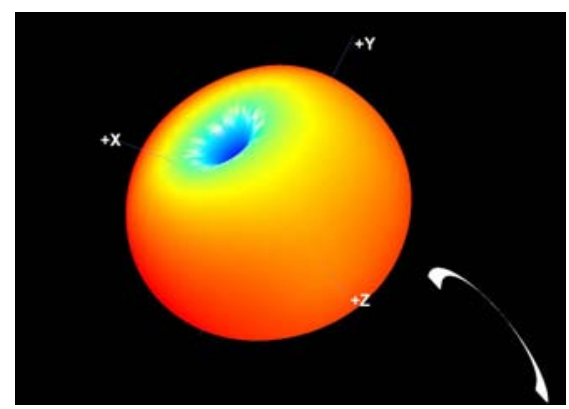

Fig 4. Measured radiation pattern of U-shaped antenna including all metal traces of the module.

\section{FABRICATION/ASSEMBLY}

Once the system topology and the antenna had been designed, the overall performance of the prototype board shown in figure 3 was verified. The complete design was then reproduced on paper using inkjet printing technology. The antenna and the circuit layout were printed and cured on paper using silver ink and the complete wireless sensor system comprising the IC (MCU+ transmitter), its discrete passive components, the temperature sensor, and a $\mathrm{Li}$-ion cell for "stand-alone" (autonomous) operation were assembled on it. The fabrication/ assembly process is outlined in this section.

\section{A. Printing}

The inkjet-printing is done in a horizontal bar by bar printing. Two different cartridge settings were used, the slow but high accurate setting for the quality of the small traces around the IC chip, and the low resolution but fast setting for the bulk printing of the UHF antenna. Resolution as low as $50 \mu \mathrm{m}$ could be achieved using the printing process [4].

\section{B. Assembly}

The assembly process on silver pads proved to be the most challenging aspect in the design process. Given the low temperature tolerance of paper and the electronic components used and the relative weaker adhesion of printed silver pads on paper, soldering had to be ruled out. Using silver epoxy by itself also proved to be a challenge due to the small size and spacing distance of $(\sim 150 \mu \mathrm{m})$ the printed silver pads on paper for the TSSOP packaged IC chip. The basic assembly was done as a 2 part process as shown in Fig.5. The first step involved holding the components in place on the printed silver pads with the use of a very small amount of adhesive between the component terminals and printed silver pads.

Once in place, conductive silver epoxy was them applied on the terminals of the components to establish a conductive path between the terminals and printed silver pads. The complete assembly of all components also had to be done using a multistep process. This was because the conductivity of the silver epoxy had a direct co-relation to its curing time and temperature, which contradicted with the limited temperature tolerance of the components used in the wireless transmitter. Due to this the assembly of components was done in the order shown in table 2 . The curing time and temperature are also shown in table 2.

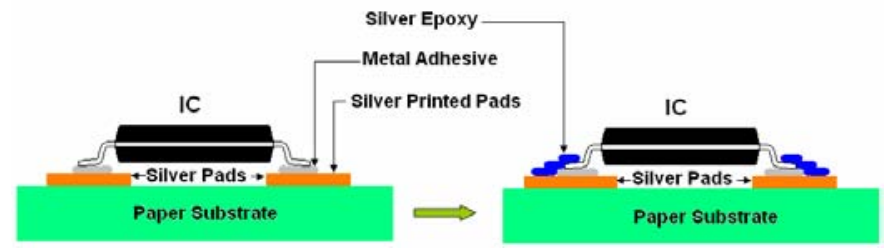

Fig 5. Assembly process for components on inkjet printed silver pads on paper substrates.

Table 2. Curing order, temperature and time used in assembly process for different components.

\begin{tabular}{|l|l|l|l|}
\hline Order & Components & $\begin{array}{l}\text { Curing } \\
\text { Temperature } \\
\left({ }^{\circ} \mathrm{C}\right)\end{array}$ & $\begin{array}{l}\text { Curing } \\
\text { Time } \\
(\text { minutes })\end{array}$ \\
\hline 1 & TC1047 Temp Sensor & 120 & 15 \\
\hline 2 & $\begin{array}{l}\text { IC, Inductors, } \\
\text { Capacitors, Resistors }\end{array}$ & 100 & 45 \\
\hline 3 & Crystal Oscillator & 80 & 90 \\
\hline 4 & ML 2020 Panasonic Cell & 60 & 240 \\
\hline
\end{tabular}

The final assembled wireless sensor prototype can be seen in figure 7. The sensor prototype was tested to study the effects of the packaging material and the series silver ink/silver epoxy interface on the sensor reading. The results can be seen in figure 6 . The measured results show good agreement with the manufacturer's specs. The relatively higher heat insulation of paper and silver ink to temperature changes compared to other conductors and packaging materials makes it an ideal substrate for housing sensors. The prototype was also tested for wireless transmission using a XR-400 RFID reader antenna interfaced to a Tektronix RSA 3408A Real Time Spectrum Analyzer (RTSA). The measured power spectrum can be seen in figure 8.

For a transmission distance of 1 feet, the measured power level emitted by the prototype was measured to be a very low $-69 \mathrm{dBm}$ at a transmission frequency of $904.4 \mathrm{MHz}$. While the measured power level does indicate that the prototype 
functions as intended at the system level, there are significant losses in the transmitted power.

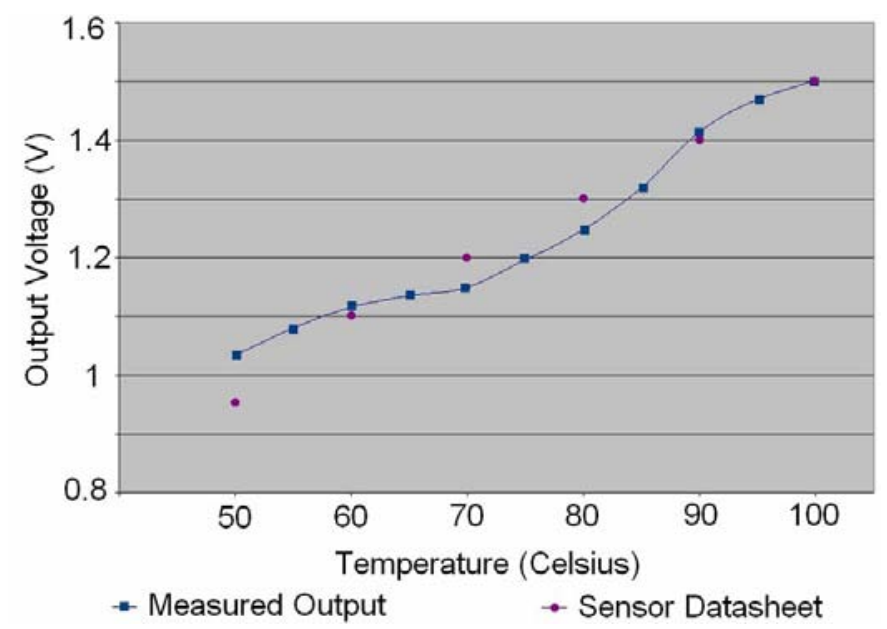

Fig 6. Temperature sensor performance. Measured vs manufacturer's specifications. .

The MSDS of the silver ink used in the printing process demonstrated a conductivity of $40 \mathrm{k}$-siemens/cm at DC [3]. However, upon measurement the DC resistance of each arm of the antenna and the power traces supplying the IC demonstrated resistances of $32 \mathrm{ohms}$ and $8 \mathrm{ohms}$ respectively. Further investigation of the cause for such large loss was found to be the printing process of the silver ink used to print the antenna and the circuit traces. Printing in the horizontal direction left striations through out the structure when observed under a microscope as shown in figure 9 .

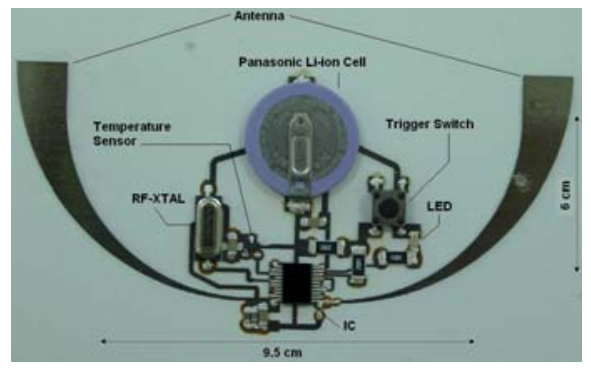

Fig 7. Wireless Sensor transmitter prototype on paper substrate using silver inkjet printing technology.

$\mathrm{AC}$ resistance, especially at $\mathrm{RF}$ frequencies, is expected to worsen due to the non-uniform surface of the structure causing not only loss in the radiating structure but also an impedance mismatch between the IC and the antenna. Increased resistance on the path from the amplifier source to the ground can also reduce the gain of the antenna and alter the optimum load impedance looking out of the power amplifier in the transmitter.

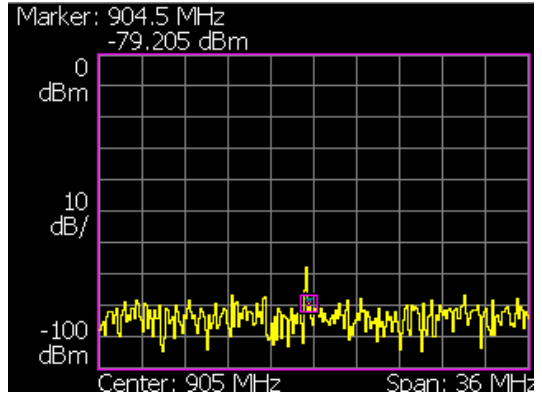

Fig 8. RTSA measured unmodulated power level emitted by the wireless sensor module.

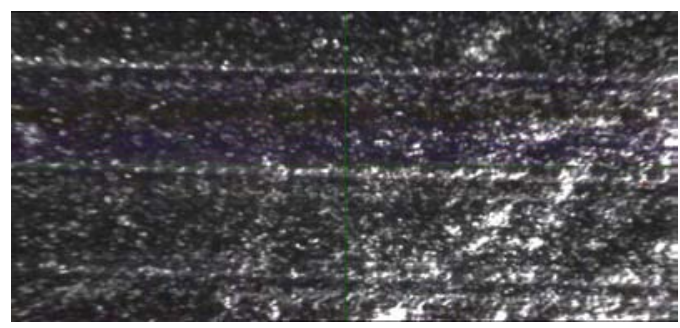

Fig 9. Surface profile of printed Antenna.

\section{CONCLUSION}

In this paper, the feasibility of inkjet printing process for the development of a "System-on-Paper" RF system was demonstrated for the first time.. The process of designing a wireless transmitting sensor module on paper was outlined and its performance was measured. While paper does offer numerous benefits as a RF packaging material, significant fundamental challenges exist with regards to the fabrication of $\mathrm{RF}$ structure on it using inkjet printing. Despite the fact that more accurate characterization of the electrical properties of conductors using inkjet printing and less stressful ways of circuit assembly on paper needs to be further investigated, the proposed approach could set the foundation for the development of ultra-low-cost "green" wireless sensors.

\section{REFERENCES}

[1] A. Rida, L. Yang, and M. M. Tentzeris, "Design and characterization of novel paper-based inkjet-printed UHF antennas for RFID and sensing applications " Procs. of the 2007 IEEE-APS Symposium, pp2749-2752, Honolulu, HI, July 2007

[2] "UHF Gen-2 System Overview". Texas Instruments, Sept 2005.

[3] Cabot Corporation, "Inkjet silver conductor AG-IJ-G-100-S1," Data Sheet, October 2006.

[4] L. Yang, A. Rida, R. Vyas, M. M. Tentzeris, "RFID Tag and RF Structures on a Paper Substrate Using Inkjet-Printing Technology," Microwave Theory and Techniques, IEEE Transactions on Volume 55, Issue 12, Part 2, Dec. 2007 Page(s):2894 - 2901 